the

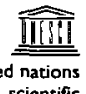

abdus salam international

and cultural
organization centre

for theoretical physics

ON BOUNDS FOR THE CHARACTERISTIC FUNCTIONS OF SOME DEGENERATE MULTIDIMENSIONAL DISTRIBUTIONS

T. Shervashidze
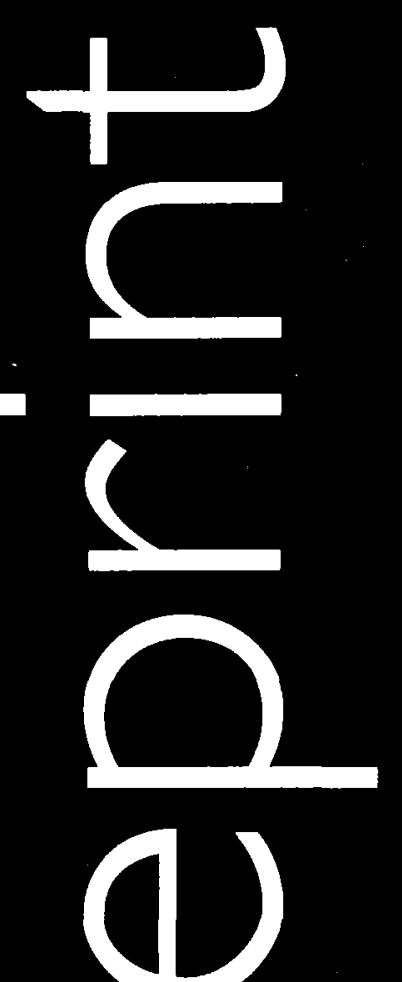

XA0300792

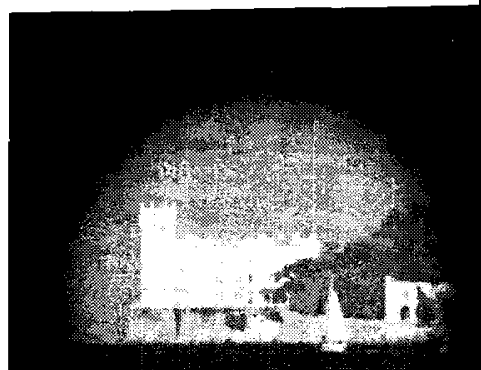

(4) 
United Nations Educational Scientific and Cultural Organization and

International Atomic Energy Agency

THE ABDUS SALAM INTERNATIONAL CENTRE FOR THEORETICAL PHYSICS

\title{
ON BOUNDS FOR THE CHARACTERISTIC FUNCTIONS OF SOME DEGENERATE MULTIDIMENSIONAL DISTRIBUTIONS
}

T. Shervashidze

A. Razmadze Mathematical Institute, Georgian Academy of Sciences, 1, M. Aleksidze St., Tbilisi 380093, Georgia and

The Abdus Salam International Centre for Theoretical Physics, Trieste, Italy.

\begin{abstract}
We discuss an application of an inequality for the modulus of the characteristic function of a system of monomials in random variables to the convergence of the density of the corresponding system of the sample mixed moments. We also consider the behavior of constants in the inequality for the characteristic function of a trigonometric analogue of the above-mentioned system when the random variables are independent and uniformly distributed. Both inequalities were derived earlier by the author from a multidimensional analogue of Vinogradov's inequality for a trigonometric integral. As a byproduct the lower bound for the spectrum of $A_{k} A_{k}^{\prime}$ is obtained, where $A_{k}$ is the matrix of coefficients of the first $k+1$ Chebyshev polynomials of first kind.
\end{abstract}

MIRAMARE - TRIESTE

December 2002 
Introduction. This note consists of two addenda to [13] which gives an upper bound for the modulus of the characteristic function (c.f.) of the system of monomials in random variables, using a multidimensional analogue [2] of the well-known inequality by I. M. Vinogradov for a trigonometric integral; see Propositions 1 and 2 below. In fact, [13] contains a more general statement for an appropriate multiple trigonometric integral, which implies another inequality for the particular random vector whose components are products of cosines of multiples of random arguments, which are independent and uniformly distributed in $[0, \pi]$; see Proposition 3 below. We refer to [13] for an answer to the question why this case is important to be considered (as well as for a short review of the problem of constructing bounds for c.f. of degenerate multidimensional distributions and a list of some basic references). Our first result concerns the constants appearing in this bound. It enables us to replace them by others which have an explicit dependence both on the number of variables and on maximal multiplicity of a random argument. It follows from the lower bound which we have constructed for the spectrum of a matrix composed by the coefficients of the Chebyshev polynomials of first kind.

On the other hand, we emphasize here the applicability of such analytic tools as the propositions mentioned, distinguishing by means of the first of them a certain class of densities such that if a population density belongs to this class, the density of a properly centered and normalized system of sample mixed moments converges to an appropriate normal density. This result is a simple consequence of the well-known limit theorems for sums of independent random vectors, but it is given just as a useful reference for statisticians, who often pose a question whether sampling distributions of parameter estimators are convergent in a stronger sense as compared with weak convergence (see, e.g., $[3,8,9,14]$ ). Another advantage is that due to the Sheffe's theorem [12] the density convergence implies convergence in variation. For some efficient examples of the application of the convergence in variation in statistics see $[10,11]$.

1. Preliminaries. Let $\xi=\left(\xi_{1}, \ldots, \xi_{s}\right) \in \mathbb{R}^{s}$ be a random vector and $f(., \zeta)$ denote the c.f. of a random vector $\zeta$ where dot stands for its argument from an Euclidean space of the same dimension as $\zeta$. Let the multiindex $\mathbf{j}=\left(j_{1}, \ldots, j_{s}\right) \neq \mathbf{0}=(0, \ldots, 0) \in \mathbb{R}^{s}$ vary in $J_{s, k}=$ $\{0,1, \ldots, k\}^{s} \backslash\{\mathbf{0}\}$ ordered lexicografically. For $\mathbf{j} \in J_{s, k}$ denote $\xi^{\mathbf{j}}=\xi_{1}^{j_{1}} \cdots \xi_{s}^{j_{s}}$ and consider the system of monomials in $s$ real variables

$$
M_{s, k}(\xi)=\left(\xi^{\mathbf{j}}, \mathbf{j} \in J_{s, k}\right) .
$$

Denote $\tau=\tau(t)=\max \left\{\left|t_{\mathbf{j}}\right|: \mathbf{j} \in J_{s, k}\right\}, \quad t=\left(t_{\mathbf{j}}, \mathbf{j} \in J_{s, k}\right) \in \mathbb{R}^{(k+1)^{s}-1}$.

If $\eta=\left(\eta_{1}, \ldots, \eta_{s}\right)$ denotes a random vector with the uniform distribution in $[0,1]^{s}$, a multidimensional analogue of Vinogradov's inequality from [2, p.39] can be written in a form

$$
\left|f\left(t, M_{s, k}(\eta)\right)\right| \leq 32^{s}(2 \pi)^{1 / k} \tau^{-1 / k} \ln ^{s-1}(2+\tau /(2 \pi)) \wedge 1 .
$$

Let $D=\left[a_{1}, b_{1}\right] \times \cdots \times\left[a_{s}, b_{s}\right]$ with positive $h_{j}=b_{j}-a_{j}<\infty, j=1, \ldots, s$; denote $D\left(j_{1}, \ldots, j_{r}\right)=\left[a_{j_{1}}, b_{j_{1}}\right] \times \cdots \times\left[a_{j_{r}}, b_{j_{r}}\right]$ and $D_{c}\left(j_{1}, \ldots, j_{r}\right)=\prod\left\{\left[a_{j}, b_{j}\right]: j=1, \ldots, s, j \neq\right.$ 
$\left.j_{1}, \ldots, j_{r}\right\}$ for $1 \leq j_{1}<\cdots<j_{r} \leq s, 1<r<s$. The notation $x\left(j_{1}, \ldots, j_{r}\right)$ and $x_{c}\left(j_{1}, \ldots, j_{r}\right)$ for $x \in \mathbb{R}^{s}$ is to be understood similarly. Denote further by $V, V_{c}\left(j_{1}, \ldots, j_{r}\right)$ the sets of vertices of the parallelepipeds $D, D_{c}\left(j_{1}, \ldots, j_{r}\right)$, respectively, and

$$
\Pi(z)=\prod_{j=1}^{r} \max \left(1,\left|z_{j}\right|\right), z=\left(z_{1}, \ldots, z_{r}\right) \in \mathbb{R}^{r}, 1 \leq r \leq s .
$$

By virtue of the fact that the inequality

$$
\tau(\widetilde{t}) \geq \prod_{j=1}^{s}\left[\min \left(1,\left|y_{j}\right|\right)\right]^{k} \tau
$$

holds for $y=\left(y_{1}, \ldots, y_{s}\right) \in \mathbb{R}^{s}$ and for $\widetilde{t}=\left(y^{\mathbf{j}} t_{\mathbf{j}}, \mathbf{j} \in J_{s, k}\right)$ and the function on the right-hand side of (1) decreases with respect to $\tau$, while the logarithm increases, we have the following assertion.

Proposition 1. If a random vector $\xi$ has the density

$$
u_{D}(x)=\left(h_{1} \cdots h_{s}\right)^{-1} \mathbb{1}_{D}(x),
$$

where $\mathbb{1}_{A}(\cdot)$ denotes the indicator of a set $A$, then the inequality

$$
\left|f\left(t, M_{s, k}(\xi)\right)\right| \leq \Pi\left(h_{1}^{-1}, \ldots, h_{s}^{-1}\right) 32^{s}(2 \pi)^{1 / k} \tau^{-1 / k} \ln ^{s-1}(2+\tau /(2 \pi)) \wedge 1
$$

holds.

Denote now by $\mathfrak{P}$ the class of probability densities $p_{\xi}(x)=p(x), x \in D$, such that $p(x)$ is continuous in $D$ and has, in $D$, continuous partial derivatives $p_{i}=p_{x_{i}}, p_{i j}=p_{x_{i} x_{j}}, \ldots, p_{1 \cdots s}=$ $p_{x_{1} \ldots x_{s}}$. Introduce the notation

$$
\begin{gathered}
C_{r}=\sum_{1 \leq j_{1}<\cdots<j_{r} \leq s} \sum_{x_{c}\left(j_{1}, \ldots, j_{r}\right) \in V_{c}\left(j_{1}, \ldots, j_{r}\right)} \Pi\left(x_{c}\left(j_{1} \ldots j_{r}\right)\right) \times \\
\times \int_{D\left(j_{1}, \ldots, j_{r}\right)} \Pi\left(x\left(j_{1}, \ldots, j_{r}\right)\right)\left|p_{j_{1} \ldots j_{r}}(x)\right| d x\left(j_{1}, \ldots, j_{r}\right), \quad 1 \leq r<s, \\
C_{0}=\sum_{x \in V} \Pi(x) p(x), C_{s}=\int_{D} \Pi(x)\left|p_{1 \ldots s}(x)\right| d x .
\end{gathered}
$$

Let $\mathfrak{P}$ also contain those densities, for which some of the edges of $D$ have an infinity length while the previous smoothness conditions are fulfilled in every bounded parallelepiped $D^{(b)} \subset D$ and the above expressions calculated for $D^{(b)}$ have finite limits (as $D^{(b)}$ approaches $D$ ) denoted again by $C_{r}, 0 \leq r \leq s$.

In [13] from (1) and Proposition 1 the following inequality is derived (for which the formula of integration by parts for several variables serves as a main tool):

$$
\left|f\left(t, M_{s, k}(\xi)\right)\right| \leq C 32^{s}(2 \pi)^{1 / k} \tau^{-1 / k} \ln ^{s-1}(2+\tau /(2 \pi)) \wedge 1
$$

where

$$
C=C\left(s, k, p_{\xi}(x)\right)=C_{0}+\cdots+C_{s}
$$

(both for the bounded and the unbounded parallelepiped $D$ ).

Of course, it is possible to consider any subsystem $m_{s, k}(\xi)$ of the system $M_{s, k}(\xi)$. The c.f. of $m_{s, k}(\xi)$ can be obtained from $f\left(t, M_{s, k}(\xi)\right)$ when substituting 0 instead of the coordinates of $t$ with the indices lying outside the set of indices corresponding to $m_{s, k}(\xi)$. Denote the suitable 
subvector of $t$ by $t_{(m)}, t_{(M)}=t$. Clearly, $\tau=\tau(t) \geq \tau\left(t_{(m)}\right)=\tau_{(m)}$ and since the right-hand side of (1) is decreasing with respect to $\tau$, we can replace $\tau$ by $\tau_{(m)}$ for the fixed $s$ and $k$. Further if $d_{(m)}=d_{(m)}(s, k)$ is the dimension of $t_{(m)}, 1 \leq d_{(m)} \leq(k+1)^{s}-1$, we have that $\tau_{(m)} \geq\left|t_{(m)}\right| d_{(m)}^{-1 / 2}$, where $\left|t_{(m)}\right|$ is the Euclidean norm of $\left|t_{(m)}\right|$, in particular, $\tau \geq|t|\left[(k+1)^{s}-1\right]^{-1 / 2}$ for $t \in \mathbb{R}^{(k+1)^{s}-1}$, and the inequalities listed can be expressed in terms of $\left|t_{(m)}\right|$ and $|t|$ as well. Let us fix the second one in terms of $t_{(m)}$ and $|t|$.

Proposition 2. If $p_{\xi}(x) \in \mathfrak{P}$, then

$$
\left|f\left(t, m_{s, k}(\xi)\right)\right| \leq C_{1}\left|t_{(m)}\right|^{-1 / k} \ln ^{s-1}\left(2+C_{2}\left|t_{(m)}\right|\right) \wedge 1
$$

where $t_{(m)} \in \mathbb{R}^{d(m)}$,

$$
\begin{gathered}
C_{1}=C\left(s, k, p_{\xi}(x)\right)=C 32^{s}(2 \pi)^{1 / k} d_{(m)}^{1 /(2 k)}, \\
C_{2}=C_{2}(s, k)=(2 \pi)^{-1} d(m)^{-1 / 2} .
\end{gathered}
$$

For $m_{s, k}(\xi)=M_{s, k}(\xi)$ we have $d_{(m)}=\left[(k+1)^{s}-1\right]$ and $t(m)=t$.

Let us now consider a trigonometric analogue of the system $M_{s, k}(\xi)$ when $\xi$ is replaced by a random vector $\eta$ uniformly distributed in $[0,1]^{s}$. Introduce the system of cosines products

$$
M_{s, k}^{(c)}(\eta)=\left(\cos j_{1} \pi \eta_{1} \cdots \cos j_{s} \pi \eta_{s}, \mathbf{j} \in J_{k, s}\right) .
$$

Performing the change of variables $\zeta_{i}=\cos \pi \eta_{i}, i=1, \ldots, s$, we arrive at the system

$$
{\widetilde{M^{(c)}}}_{s, k}(\zeta)=\left(T_{j_{1}}\left(\zeta_{1}\right) \cdots T_{j_{s}}\left(\zeta_{s}\right), \mathbf{j} \in J_{s, k}\right)
$$

where $T_{r}(x)=\sum_{j=0}^{r} a_{r j} u^{j}, u \in \mathbb{R}^{1}$, is the Chebyshev polynomial of first kind and $\zeta=\left(\zeta_{1}, \ldots, \zeta_{s}\right)$ is a random vector with the density function

$$
q(y)=\pi^{-s} \prod_{j=1}^{s}\left(1-y_{j}^{2}\right)^{-1 / 2} \mathbb{1}_{(-1,1)^{s}} .
$$

Let $A_{k}$ be the $(k+1) \times(k+1)$-matrix with rows $\left(a_{r 0}, \ldots, a_{r r}, 0, \ldots, 0\right), r=0, \ldots, k$, and denote by $\lambda_{k}$ the least eigenvalue of the matrix $A_{k} A_{k}^{\prime}$.

Proposition 2 is not directly applicable to the system of polynomials $\widetilde{M^{(c)}} s, k$, but in [13] this case was treated using some truncation argument. As a result the following inequality has been proved which has turned out to have an optimal exponent of $|t|$ : for $s=1$ there exist directions in $\mathbb{R}^{k}$ along which the c.f. modulus behaves asymptotically as $|t|^{-1 /(2 k)}$.

Proposition 3. The following inequality holds:

$$
\left|f\left(t, M_{s, k}^{(c)}(\eta)\right)\right| \leq C_{3}|t|^{-\frac{1}{k(s+1)}} \ln ^{\frac{s-1}{s+1}}\left(2+C_{4}|t|\right) \wedge 1
$$

where

$$
C_{3}=C_{3}(s, k)=C_{3}^{0}(s, k) \lambda_{k}^{-\frac{1}{2 k(s+1)}}, C_{4}=C_{4}(s, k)=C_{4}^{0}(s, k) \lambda_{k}^{s / 2}
$$

with

$$
C_{3}^{0}=2^{\frac{9 k s+1}{k(s+1)}} \pi^{-\frac{2 k s-1}{k(s+1)}} s^{\frac{1}{s+1}}\left[(k+1)^{s}-1\right]^{\frac{1}{2 k(s+1)}}
$$




$$
C_{4}^{0}(s, k)=C_{2}(s, k)=(2 \pi)^{-1}\left[(k+1)^{s}-1\right]^{-1 / 2} .
$$

To have a complete picture of dependence of the constants $C_{3}(s, k)$ and $C_{4}(s, k)$ on $k$ and $s$ we need to estimate $\lambda_{k}$ from below.

\section{Bounds for $\lambda_{k}$.We have}

$$
\lambda_{k}=\lambda_{\min }\left(B_{k}\right),
$$

where $B_{k}=A_{k} A_{k}^{\prime}$ and $A_{k}$ as introduced below is the lower triangular matrix of coefficients of the Chebyshev polynomials

$$
T_{n}(x)=\cos n \arccos x=\sum_{i=0}^{n} a_{n i} x^{i}, n=0,1, \ldots, k .
$$

As it is well-known (see, e.g., $[4]$, p.25),

$$
T_{n+1}(u)=2 u T_{n}(u)-T_{n-1}(u), n \geq 1, T_{0}(u) \equiv 1, T_{-1}(u) \equiv 0,
$$

which implies that

$$
a_{n+1, i}=2 a_{n, i-1}-a_{n-1, i}, 0 \leq i \leq n .
$$

Further, $a_{n i}=0, i>n, a_{n 0}=\cos \frac{n \pi}{2}, a_{00}=1, a_{n+1, n+1}=2 a_{n n}-a_{n-1, n}=2 a_{n n}$, i.e., $a_{n n}=2^{n}$, $n \geq 0$, and therefore $\left|A_{k}\right|=1 \cdot 2 \cdots 2^{k}=2^{\frac{k(k+1)}{2}} ;$ thus

$$
\left|B_{k}\right|=\left|A_{k} A_{k}^{\prime}\right|=2^{k(k+1)},
$$

which gives that $\lambda_{k}^{k+1} \leq 2^{k(k+1)}$ and

$$
\lambda_{k} \leq 2^{k}=\lambda_{k}^{*} .
$$

To obtain a lower bound for $\lambda_{k}$ let us estimate the trace of $B_{k}^{-1}$ from above. If $\lambda_{0} \geq \cdots \geq \lambda_{k}$ stand for the eigenvalues of $B_{k}$ in decreasing order, $\lambda_{j}^{-1}, j=0, \ldots, k$, are the eigenvalues of $B_{k}^{-1}$ in increasing order and

$$
\frac{1}{\lambda_{k}} \leq \frac{1}{\lambda_{0}}+\cdots+\frac{1}{\lambda_{k}}=\sum_{j=0}^{k} \frac{\left|B_{j j}\right|}{\left|B_{k}\right|} \leq\left|B_{k}\right|^{-1} \sum_{j=0}^{k} \frac{b_{00} \cdots b_{k k}}{b_{j j}}
$$

where $b_{j j}, j=0, \ldots, k$, are diagonal elements of the positive definite matrix $B_{k}$ and $\left|B_{j j}\right|$ are principal minors of $\left|B_{k}\right|$.

Let us estimate $b_{n n}$ from above. From (2) we have that

$$
a_{n+1, i}^{2}=4 a_{n, i-1}^{2}+a_{n-1, i}^{2}-4 a_{n, i-1} a_{n-1, i}
$$

and summing (5) with respect to $i$ from 1 till $n+1$, we obtain

$$
b_{n+1, n+1}-\cos ^{2}(n+1) \frac{\pi}{2}=4 b_{n n}+b_{n-1, n-1}-\cos ^{2}(n-1) \frac{\pi}{2}-4 \sum_{i=1}^{n+1} a_{n, i-1} a_{n-1, i} .
$$

The Cauchy inequality leads to the estimate

$$
\left(\sum_{i=1}^{n+1} a_{n, i-1} a_{n-1, i}\right)^{2} \leq \sum_{i=1}^{n+1} a_{n, i-1}^{2} \sum_{i=1}^{n+1} a_{n-1, i}^{2}=b_{n n}\left(b_{n-1, n-1}-\cos ^{2}(n-1) \pi / 2\right),
$$


whence

$$
b_{n+1, n+1} \leq\left(2 \sqrt{b_{n n}}+\sqrt{b_{n-1, n-1}}\right)^{2} .
$$

Let us now introduce a new sequence $s_{n}$ such that $b_{n n} \leq s_{n}^{2}$ for $n \geq 0, s_{0}=b_{00}=1$, $s_{1}=\sqrt{b_{11}}=2$ and

$$
s_{n+1}=2 s_{n}+s_{n-1} \text {. }
$$

The sequence $\left\{s_{n}\right\}$ satisfying (6) must be of the type

$$
s_{n}=c_{1} \mu_{1}^{n}+c_{2} \mu_{2}^{n},
$$

where $\mu_{1}, \mu_{2}$ are the roots of the characteristic equation

$$
\mu^{2}-2 \mu-1=0
$$

i.e., $\mu_{1}=1+\sqrt{2}, \mu_{2}=1-\sqrt{2}$, and the constants $c_{1}, c_{2}$ are calculated by the initial conditions $s_{0}=1$ and $s_{1}=2: c_{1}=\frac{\sqrt{2}+1}{2 \sqrt{2}}, c_{2}=\frac{\sqrt{2}-1}{2 \sqrt{2}}$.

Therefore

$$
s_{n}=\frac{1}{2 \sqrt{2}}\left((\sqrt{2}+1)^{n+1}-(1-\sqrt{2})^{n+1}\right)
$$

(in particular, we have $s_{0}=1, s_{1}=2, s_{2}=5$ ).

It is easy to see that

$$
\begin{aligned}
s_{n}^{2} & =\frac{1}{8}\left[(\sqrt{2}+1)^{2(n+1)}+(1-\sqrt{2})^{2(n+1)}+2(-1)^{n}\right]= \\
& =\frac{1}{8}\left[(3+2 \sqrt{2})(\sqrt{2}+1)^{2 n}+(3-2 \sqrt{2})^{n+1}+(-1)^{n}\right]<6^{n} .
\end{aligned}
$$

Finally

$$
\lambda_{k}^{-1} \leq 2^{-k(k+1)} \sum_{j=0}^{k} 6^{-j} \cdot 6^{\sum_{j=0}^{k} j}<2^{-k(k+1)} 6^{\frac{k(k+1)}{2}} \frac{1}{1-1 / 6}=\frac{6}{5}\left(\frac{3}{2}\right)^{\frac{k(k+1)}{2}}
$$

and we arrive at the following

Proposition 4. A lower bound of the spectrum of matrix $A_{k} A_{k}^{\prime}$ is given by the relation

$$
\lambda_{k} \geq \frac{5}{6}\left(\frac{2}{3}\right)^{\frac{k(k+1)}{2}}=\lambda_{k *}
$$

In particular, $\lambda_{0 *}=\frac{5}{6}, \lambda_{1 *}=\frac{5}{9}, \lambda_{2 *}=\frac{10}{27}$.

Thus in the constants $C_{3}$ and $C_{4}$ appearing in Proposition 3 one can replace $\lambda_{k}$ by $\lambda_{k *}$ defined in (7).

Let us now compare $\lambda_{k}$ with $\lambda_{k *}$ for $k=0,1,2$.

For $k=0 \quad B_{0}=1, \lambda_{0}=1, \lambda_{0 *}=\frac{5}{6}$,

for $k=1 \quad B_{1}=\left(\begin{array}{cc}1 & 0 \\ 0 & 4\end{array}\right), \lambda_{1}=1, \lambda_{1 *}=\frac{5}{9}$,

for $k=2 \quad B_{2}=\left(\begin{array}{ccc}1 & 0 & -1 \\ 0 & 4 & 0 \\ -1 & 0 & 17\end{array}\right), \lambda_{2}=9-\sqrt{68}=0.73, \lambda_{2 *}=\frac{10}{27}$. 
3. Convergence of the Density of a System of Sample Mixed Moments. Proposition 2 finds a convenient application to obtain a limit theorem for the density of the system of sample mixed moments. The background for it is the multidimensional version of Gnedenko's famous local limit theorem for densities of sums of independent identically distributed random variables with finite variances due to Hekendorf [6], which asserts that integrability of some natural power of the modulus of c.f. of common distribution is necessary and sufficient for the uniform convergence of densities of sums to the normal density.

Let us first consider the case of one-dimensional population $\xi$ with a density $p(x), x \in \mathbb{R}^{1}$, such that $\max (1,|x|)\left|p^{\prime}(x)\right| \in L\left(\mathbb{R}^{1}\right)$ and there exist limits of $x p(x)$ as $x$ tends to infinite ends of an interval supporting $p(x)$, i.e., $p(x) \in \mathfrak{P}$.

In this case $M_{1, k}(\xi)=\left(\xi, \ldots, \xi^{k}\right), k>1$, and according to Proposition 2,

$$
\left|f\left(t, M_{1, k}(\xi)\right)\right| \leq C_{1}(k, p(x))|t|^{-1 / k} \wedge 1, t \in \mathbb{R}^{k} .
$$

If $\mathbf{E} \xi^{2 k}<\infty$, the random vector $M_{1, k}(\xi)$ obeys the covariance matrix

$$
\mathbf{C}=\left(\mathbf{c}_{i j}=\mathbf{E} \xi^{i+j}-\mathbf{E} \xi^{i} \mathbf{E} \xi^{j}, i, j=1, \ldots, k\right)
$$

which is nonsingular since the inequality

$$
\mathbf{E}\left[\sum_{j=1}^{k} a_{j}\left(\xi^{j}-\mathbf{E} \xi^{j}\right)\right]^{2}=\int_{\mathbb{R}^{1}}\left[\sum_{j=1}^{k} a_{j}\left(x^{j}-\mathbf{E} \xi^{j}\right)\right]^{2} p(x) d x>0,
$$

holds true for any $a=\left(a_{1}, \ldots, a_{k}\right) \in \mathbb{R}^{k}$ except for $a=0$.

Under the condition $\mathbf{E} \xi^{2 k}<\infty$ the Lindeberg-Lévy theorem implies that if $\xi^{(1)}, \xi^{(2)}, \ldots$ are independent copies of the random variable $\xi$, then the normalized sum

$$
S_{n}=n^{-1 / 2}\left[M_{1, k}\left(\xi^{(1)}+\cdots+M_{1, k}\left(\xi^{(n)}\right)-n \mathbf{E} M_{1, k}\left(\xi^{(1)}\right)\right]\right.
$$

as $n \rightarrow \infty$ has the limiting normal distribution $\Phi_{\mathbf{C}}$ with mean 0 and covariance matrix $\mathbf{C}$. If we now introduce the notation

$$
\overline{M_{1, k}(\xi)}=\frac{1}{n} \sum_{j=1}^{n} M_{1, k}\left(\xi^{(j)}\right)
$$

for the vector of sample initial moments constructed by the sample $\xi^{(1)}, \ldots, \xi^{(n)}$ from the population $\xi$ with the density $p(x)$, then the normalized sum $S_{n}$ can be written as

$$
S_{n}=\sqrt{n}\left[\overline{M_{1, k}(\xi)}-\mathbf{E} M_{1, k}\left(\xi^{(1)}\right)\right]
$$

and we obtain the assertion about the weak limit $\Phi_{C}$ for the distribution $P_{S_{n}}$ of the system of sample initial moments with the suitable centering and normalization.

But for those $n$ for which $P_{S_{n}}$ obeys the density $p_{S_{n}}(x)$ with respect to the Lebesgue measure in $\mathbb{R}^{k}$ we have the following expression for the variation distance between $P_{S_{n}}$ and $\Phi_{\mathbf{C}}$, i.e. , for the value in $\mathbb{R}^{k}$ of the complete variation of the signed measure $P_{S_{n}}-\Phi_{\mathbf{C}}$,

$$
\left\|P_{S_{n}}-\Phi_{\mathbf{C}}\right\|=\int_{\mathbb{R}^{k}}\left|p_{S_{n}}(x)-\varphi_{\mathbf{C}}(x)\right| d x
$$


which according to Scheffé's theorem [12] tends to zero if densities converge pointwise. So the conditions guaranteeing the density convergence imply the convergence in variation. As we have already mentioned, the density convergence in our situation is equivalent to the existence of a natural number $r$ such that $\left|f\left(t, M_{1, k}(\xi)\right)\right|^{r} \in L\left(\mathbb{R}^{k}\right)$. Let us try to find $r$.

Using (8) and passing to the polar coordinates, we formally have

$$
\begin{gathered}
\int_{\mathbb{R}^{k}}\left|f\left(t, M_{1, k}(\xi)\right)\right|^{r} d t \leq V(B)+C_{1} \int_{\mathbb{R}^{k} \backslash B}|t|^{-r / k} d t \\
\leq V(B)+C_{1}^{\prime} \int_{b}^{\infty}|t|^{k-1} \cdot|t|^{-r / k} d|t|
\end{gathered}
$$

where $B$ is the ball of a radius $b$ in $\mathbb{R}^{k}$ centered at the origin outside which the inequality (8) becomes non-trivial and $V(B)$ is its volume, while $C_{1}^{\prime}$ is the product of $C_{1}$ and the integral taken from the factor of Jacobian depending on angle coordinates. The latter integral is finite if $-\frac{r}{k}+k-1<-1$ and hence

$$
r>k^{2}
$$

e.g., $r$ can be taken equal to $k^{2}+1$.

It is evident that uniform, exponential and normal densities belong to the class $\mathfrak{P}$ introduced above.

Example 1. Let $\xi$ have the uniform distribution in $[0,1]$. Consider the random vector $M_{1,2}(\xi)=\left(\xi, \xi^{2}\right)$ and let $\xi^{(1)}, \xi^{(2)}, \ldots$ be independent copies of the random variable $\xi$. As $\mathbf{E} \xi^{n}=\frac{1}{n+1}, n=1,2, \ldots$, we have $\mathbf{E} M_{1,2}(\xi)=(1 / 2,1 / 3)$,

$$
\mathbf{C}=\left(\begin{array}{ll}
1 / 12 & 1 / 12 \\
1 / 12 & 4 / 45
\end{array}\right)
$$

and, according to (10), $\left|f\left(t, M_{1,2}(\xi)\right)\right|^{r}$ is integrable for $r=5$. Thus for $n \geq 5$ there exists a density of $S_{n}=\sqrt{n}\left(\overline{M_{1,2}(\xi)}-(1 / 2,1 / 3)\right)$ which converges to $\varphi_{\mathbf{C}}(x)$ in $\mathbb{R}^{2}$ and as a result $\left\|P_{S_{n}}-\Phi_{\mathbf{C}}\right\| \rightarrow 0 \quad(n \rightarrow \infty)$.

Example 2. Let $\xi$ have the exponential distribution with parameter 1. According to Proposition 2 the random vector $M_{1,2}(\xi)$ has the c.f. integrable in 5 th power and as $\mathbf{E} \xi^{n}=n$ ! the random vector $S_{n}=\sqrt{n}\left(\overline{M_{1,2}(\xi)}-(1,2)\right)$ constructed by independent copies $\xi^{(1)}, \ldots, \xi^{(n)}$ of $\xi$ has a limiting normal density $\varphi_{\mathbf{C}}(x)$ with

$$
\mathbf{C}=\left(\begin{array}{cc}
1 & 4 \\
4 & 20
\end{array}\right)
$$

as a result $\left\|P_{S_{n}}-\Phi_{\mathbf{C}}\right\| \rightarrow 0(n \rightarrow \infty)$.

If $s>1$ and the population $\xi=\left(\xi_{1}, \ldots, \xi_{s}\right)$ has a density $p(x) \in \mathfrak{P}$, then the c.f. of the system of monomials $M_{s, k}(\xi)$ behaves according to Proposition 3, i.e., passing again to the polar coordinates, we obtain that its $r$ th power is integrable as a function of $t \in \mathbb{R}^{(k+1)^{s}-1}$ as soon as the function

$$
|t|^{(k+1)^{s}-2}|t|^{-r / k} \ln _{8}^{r(s-1)}\left(2+C_{2}(k, s)|t|\right) \in L((b, \infty))
$$


for some positive $b$. But it takes place if $(k+1)^{s}-2-r / k<-1$, and finally for $r$ we have the inequality

$$
r>k\left[(k+1)^{s}-1\right]
$$

which reduces to (10) for $s=1$.

Let $\mathbf{E}\left(\xi_{1} \cdots \xi_{s}\right)^{2 k}<\infty$, then the covariance matrix of the random vector $M_{s, k}(\xi)$ is as follows:

$$
\mathbf{C}=\left(\mathbf{c}_{\mathrm{ij}}=\mathbf{E} \xi^{\mathbf{i}+\mathbf{j}}-\mathbf{E} \xi^{\mathbf{i}} \mathbf{E} \xi^{\mathbf{j}}, \mathbf{i}, \mathbf{j} \in J_{s, k}\right),
$$

and its nonsingularity proved as in (9) for $s=1$.

If now we introduce the system of sample mixed moments constructed by independent copies of $\xi=\left(\xi_{1}, \ldots, \xi_{s}\right)$ denoted by $\xi^{(1)}, \ldots, \xi^{(n)}$ as

$$
\overline{M_{s, k}(\xi)}=\frac{1}{n} \sum_{j=1}^{n} M_{s, k}\left(\xi^{(j)}\right)
$$

and note that according to the Lindeberg-Lévy theorem

$$
S_{n}=n^{-1 / 2}\left[M_{s, k}\left(\xi^{(1)}+\cdots+M_{s, k}\left(\xi^{(n)}\right)-n \mathbf{E} M_{s, k}\left(\xi^{(1)}\right)\right]\right.
$$

has a weak limit $\Phi_{\mathbf{C}}$ in $\mathbb{R}^{(k+1)^{s}-1}$, then the following proposition can be derived from Hekendorf's theorem, Proposition 2 and (11).

Proposition 5. If an s-dimensional population $\xi$ has a density $p(x) \in \mathfrak{P}$ and $\mathbf{E}\left(\xi_{1} \cdots \xi_{s}\right)^{2 k}<$ $\infty$, then the system of sample mixed moments, constructed by $n$ independent observations transformed by proper centering and norming into

$$
S_{n}=\sqrt{n}\left[\overline{M_{s, k}(\xi)}-\mathbf{E} M_{s, k}\left(\xi^{(1)}\right)\right],
$$

has a density $p_{S_{n}}(x)$ as soon as $n$ exceeds the lower bound defined by (11) which uniformly converges to the normal density $\varphi_{\mathbf{C}}(x)$ in $\mathbb{R}^{(k+1)^{s}-1}$ and as a consequence the convergence in variation $\left\|P_{S_{n}}-\Phi_{\mathbf{C}}\right\| \rightarrow 0(n \rightarrow \infty)$ holds too.

Example 3. Let $\xi=\left(\xi_{1}, \xi_{2}\right)$ have the normal distribution with the zero mean and unit variances and covariance $\rho, \rho^{2}<1$. According to Proposition 2 the random vector $M_{2,1}(\xi)=$ $\left(\xi_{2}, \xi_{1}, \xi_{1} \xi_{2}\right)$ has the c.f. integrable in 4 th power, and random vector $S_{n}=\sqrt{n}\left(\overline{M_{2,1}(\xi)}-(0,0, \rho)\right)$ constructed by independent copies $\xi^{(1)}, \ldots, \xi^{(n)}$ of $\xi$ has the limiting normal density $\varphi_{\mathbf{C}}(x)$ with

$$
\mathbf{C}=\left(\begin{array}{ccc}
1 & \rho & 0 \\
\rho & 1 & 0 \\
0 & 0 & 1+\rho^{2}
\end{array}\right) ;
$$

as a consequence $\left\|P_{S_{n}}-\Phi_{\mathbf{C}}\right\| \rightarrow 0(n \rightarrow \infty)$.

According to the properties of variation distance the convergence in variation asserted by Proposition 5 is preserved for any measurable function of the random vector $S_{n}$. Thus it remains valid, e.g., for any system of sample central moments. As there exist no results of Slutsky type(asserting that the limiting distribution of a sequense of random variables is preserved when any even dependent sequence is added to the initial one which tends to zero in probability; see, 
e.g.,[5]) for densities, one should use the theorem on continuously differentiable mapping (see Theorem 4.2.5 in [1]) to calculate the parameters of the limiting normal distribution. Using the latter theorem again we arrive at the covariance matrix $\mathbf{C}$ of the limiting (in the sense of convergence in variation) normal distribution of the properly centered and normed vector composed of the sample coefficients of skewness and curtosis constructed by means of independent copies $\xi^{(1)}, \ldots, \xi^{(n)}$ of a random variable $\xi$ with finite eighth moment. If $\xi$ is normal, then

$$
\mathbf{C}=\left(\begin{array}{cc}
6 & 0 \\
0 & 33
\end{array}\right)
$$

Although convergence in variation of the distribution of multivariate measure of curtosis seems difficult to treat by means of similar arguments as completely as the weak convergence is treated in [7], the joint distribution of sample marginal measures of skewness and curtosis could be considered definitely.

\section{ACKNOWLEDGEMENTS}

The author is grateful to the Abdus Salam International Centre for Theoretical Physics, Trieste, Italy, for the possibility to visit the Centre for three months during which this work was carried out, as well as to the University of Rome "La Sapienza" for the invitation initiated by Prof. P. E. Ricci and useful discussions of the above results at the seminar held there.

\section{REFERENCES}

1.T. W. Anderson, An introduction to multivariate statistical analysis. Chapman and Hall, New York etc.,1958.

2. G. I. Arkhipov, A. A. Karatsuba, and V. N. Chubarikov, The theory of multiple trigonometric sums. (Russian) Nauka, Moscow, 1987.

3. D.G. Boos, A converse to Scheffé's theorem. Ann. Statist., 13(1985), No. 1, 423-427.

4.T. S. Chihara, An introduction to orthgonal polynomials. Gordon and Breach, New York etc.,1978.

5. H. Cramér, Mathematical methods of statistics. Princeton University Press, Princeton, 1946, 1999.

6. H. Hekendorf, A higher-dimensional limit theorem for distribution functions. Ukrain. Mat. Zh., 16(1964), 353-373..

7. N. Henze, The asymptotic behaviour of a variant of multivariate curtosis. Commun. Statist.-Theory Meth., 23(1994), 1047-1061.

8. P. Jeganathan, Strong convergence of distributions of estimators. Ann. Statist., 15(1987), No. $4,1699-1708$.

9. P. Jeganathan, On the strong approximation of the distributions of estimators in linear stochastic models, I and II: Stationary and explosive models. Ann. Statist., 16(1988), No. 3, $1283-1314$. 
10. E. V. Khmaladze, Estimation of the necessary number of observations for discriminating simple close alternatives.(Russian) Teoriya Veroyatn. i Primenen., 20(1975), No. 1,115-125; English transl.: Theory Probab. Appl., 20(1975), 116-126.

11. E. V. Khmaladze, Goodness of fit tests and scanning innovation martingales. Ann. Statist., 21(1993), No.2, 798-829.

12. H. Scheffé, A useful convergence theorem for probability distributions. Ann. Math. Statist.,18(1947), No.3, 434-438.

13. T. Shervashidze, Bounds for the characteristic functions of the system of monomials in random variables and of its trigonometric analogue.Georgian Math.J. 4(1997), No. 6, 579-584.

14. T. J. Sweeting, On a converse to Scheffé's theorem. Ann. Statist., 14(1986), No.3, 1252-1256. 